\title{
Hipertensión pulmonar en paciente VIH positiva.
}

\author{
Rafael Silva, ${ }^{1,2,6}$ Claudio Pacheco, ${ }^{1,3}$ Rodrigo Vega, ${ }^{4}$ Macarena Maza, ${ }^{5}$ Felipe Águila. ${ }^{5}$
}

1.- Servicio de Medicina Hospital Regional de Talca.
2.- Unidad de Enfermedades Respiratorias Hospital Regional de Talca.
3.- Unidad de Cardiología Hospital Regional de Talca
4.- Servicio de Radiología Hospital Regional de Talca
5.- Servicio de Urgencia Hospital Regional de Talca
6.- Facultad de Ciencias de la Salud, Universidad Autónoma de Chile
Resumen: Se presenta el caso clínico de una paciente de 30 años, con diagnóstico de VIH + desde el año 2000, en terapia antirretroviral desde el año 2004. Desde el año 2007 con disnea de esfuerzo progresiva, que en el último año se hace severa, asociada a astenia, fatigabilidad y edema vespertino de extremidades inferiores. El ecocardiograma detecta un aumento de la presión de arteria pulmonar, lo que se confirma a través de un cateterismo cardíaco derecho. Se descarta tromboembolismo pulmonar y se concluye como una HAP secundaria a VIH.

La hipertensión arterial pulmonar (HAP) es una enfermedad de baja frecuencia y se puede asociar a infección por virus de la inmunodeficiencia humana (VIH), presentando una prevalencia 6 a 12 veces mayor en relación a individuos sin infección por VIH. La HAP es independiente del recuento de linfocitos T CD4 y en su patogenia participan proteínas virales, como la glicoproteína 120 y las proteínas Nef y Tat, que estimulan una cascada inflamatoria sistémica, induciendo angiogénesis en los vasos pulmonares. El estudio hemodinámico a través de cateterismo derecho es el método de elección para la confirmación diagnóstica. 


\section{Abstract:}

We report the case of a patient 30 years, diagnosed with human immunodeficiency virus (HIV) since 2000, on antiretroviral therapy since 2004. Since 2007 with progressive dyspnea, which in the past year becomes severe, associated with asthenia, fatigue and edema of lower limbs evening. Echocardiography detects an increase in pulmonary artery pressure, which is confirmed by a right heart catheterization. Pulmonary embolism is ruled out and concludes as a pulmonary arterial hypertension (PAH) secondary to HIV.

Pulmonary arterial hypertension is a disease of low

\section{Introducción:}

La hipertensión arterial pulmonar (HAP) es una enfermedad de baja frecuencia, con síntomas poco específi$\cos$, tales como fatiga y disnea leve frente a ejercicio ${ }^{1} \mathrm{y}$ que se puede asociar a múltiples situaciones clínicas ${ }^{2}$. Por otro lado, en pacientes portadores del virus de la inmunodeficiencia humana (VIH), se han descrito complicaciones cardiovasculares, tales como pericarditis, miocarditis, cardiomiopatía dilatada, endocarditis, enfermedad de arterias coronarias, neoplasias e hipertensión arterial pulmonar ${ }^{3}$. Esta última, denominada Hipertensión pulmonar asociada a VIH (HTP-VIH) ha sido poco detectada en las décadas anteriores por el foco en los aspectos infecciosos de esta enfermedad ${ }^{4}$ En estos pacientes se encuentra un aumento de la presión arterial pulmonar media, mayor o igual a $25 \mathrm{~mm} /$ $\mathrm{Hg}$ en reposo, situación detectada por monitoreo hemodinámico realizado por cateterismo cardíaco derecho (CCD). ${ }^{5}$

La incidencia de HTP-VIH se estima en 1/200 pacientes VIH (+), a diferencia de la incidencia de HAP de $1 / 200000$ encontrada en la población general ${ }^{6}$. En un estudio prospectivo que incluyó a 7648 adultos VIH (+), se encontró una prevalencia de HAP de $0,46 \%$, dato idéntico al descrito en 1990 , cuando se conocieron los primeros estudios de esta asociación ${ }^{8}$.

Se presenta caso clínico de paciente portadora de VIH en tratamiento antiviral en la cual se detecta HTP-VIH.

\section{Caso clínico:}

Paciente de 30 años, sexo femenino portadora de VIH frequency and can be associated with infection by HIV, with prevalence 6 to 12 times greater relative to individuals without HIV infection. PAH is independent of CD4 T lymphocyte count and viral proteins involved pathogenesis, such as glycoprotein 120 and Nef and Tat proteins, which stimulate a systemic inflammatory cascade, inducing angiogenesis in the pulmonary vessels. The hemodynamic study through right heart catheterization is the method of choice for diagnostic confirmation.

Key words: HIV- related pulmonary hypertension, primary pulmonary hypertension, human immunodeficiency virus

(+) diagnosticado el año 2000, en terapia antirretroviral desde el año 2004, actualmente con neviparina 200 mg.cada12 hrs. y bacavir/lamivudina 600/300 mg. / día. Tiene antecedentes de tuberculosis pulmonar a los 16 años, tratada, sin secuelas pulmonares posteriores y de consumir 30 paquetes cigarrillos/año, hábito suspendido el año 2010, con espirometria actual normal. No usa anticonceptivos orales.

Relata disnea desde el año 2007, que en el último año aumenta de intensidad, siendo actualmente de pequeños esfuerzos, más astenia, fatiga muscular y edema vespertino de extremidades inferiores. Sin episodios sincopales.

Se hospitaliza en junio de 2010 por una neumonía comunitaria bilateral realizando tratamiento con Ceftriaxona 1 gramo endovenoso cada 12 horas por 10 días, con buena respuesta clínica. $\mathrm{Al}$ examen físico cardíaco se detecta soplo holo-sistólico IV/VI en borde para esternal derecho, que aumenta en inspiración. Se realiza eco cardiograma que muestra insuficiencia severa de la válvula tricúspide, calculándose una presión arteria pulmonar de $100 \mathrm{mmHg}$, según reflujo de válvula tricúspide, con un ventrículo derecho de $48 \mathrm{~mm}$.de grosor de pared y una fracción de eyección de $67 \%$.

Se constata eco dúplex de extremidades inferiores normal y una angiografía arterial pulmonar por tomografía computada multicorte (angio TAC de pulmón) sin evidencia de tromboembolismo pulmonar, pero con signos indirectos de HAP (Figura 1).

Se efectúa CCD con prueba de nitroprusiato (Tabla 1) que constata presión de arteria pulmonar elevada, no modificable con vasodilatadores, pero si presenta un 
aumento del gasto cardíaco en dicha prueba. Se agrega al tratamiento diltiazem $60 \mathrm{mg}$ c/12 horas vía oral.

Se interpreta el cuadro como hipertensión pulmonar secundario a VIH positivo.

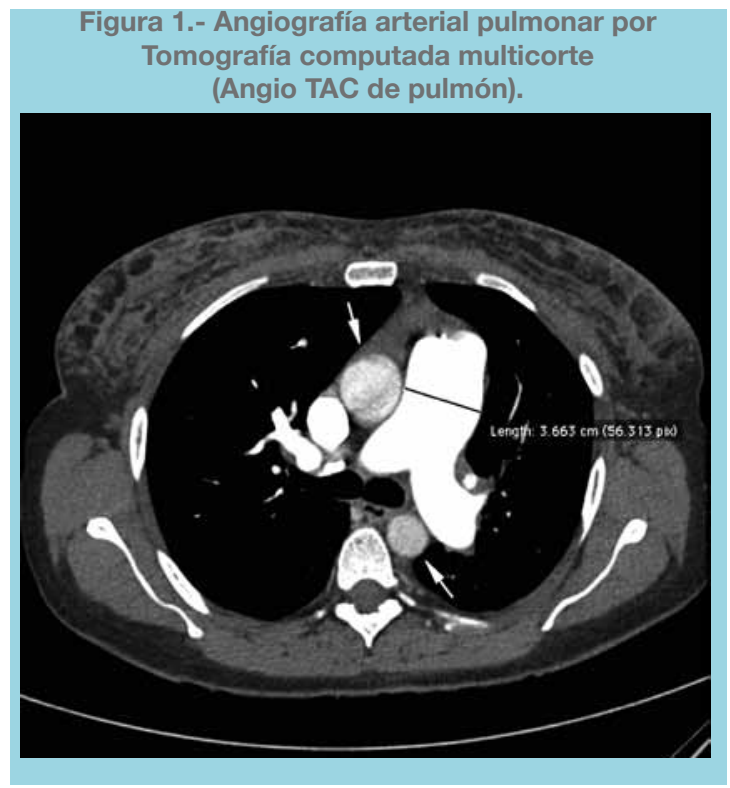

Corte axial muestra aumento de diámetro transversal del tronco de arteria pulmonar (línea negra) y aumento de tamaño transversal de arteria pulmonar en comparación con diámetro transversal de aorta ascendente y descendente al mismo nivel (flechas blancas).

Tabla 1 - Estudio hemodinámico con prueba de nitroprusiato de sodio. (Entre paréntesis dosis absolutas).

\begin{tabular}{|c|c|c|c|c|c|c|c|}
\hline $\begin{array}{l}\text { Dosis de } \\
\text { nitroprusiato } \\
\text { de sodio }\end{array}$ & $\begin{array}{l}\text { Hora } \\
\text { Adminis- } \\
\text { tración }\end{array}$ & FC & $\begin{array}{l}\text { FC PA no } \\
\text { invasiva }\end{array}$ & $\begin{array}{c}\text { PAM no } \\
\text { invasiva } \\
\text {-PAM } \\
\text { pulmonar }\end{array}$ & $\begin{array}{c}\text { PA } \\
\text { Pulmonar }\end{array}$ & $\begin{array}{l}\text { Presión } \\
\text { capilar } \\
\text { pulmonar }\end{array}$ & $\begin{array}{c}\text { Gasto } \\
\text { cardiaco }\end{array}$ \\
\hline Basal & $11: 31$ & 80 & $108 / 78$ & $50-38$ & $88 / 45$ & $26 / 15$ (19) & \\
\hline $0,5 \mu \mathrm{g} / \mathrm{kg} / \mathrm{min}(9)$ & $11: 51$ & 90 & $107 / 81$ & $54-45$ & $90 / 46$ & - & 1,6 \\
\hline $1 \mu \mathrm{g} / \mathrm{kg} / \min (18)$ & 11:56 & 93 & $99 / 74$ & $71-37$ & $82 / 48$ & $43 / 30(34)$ & 2,0 \\
\hline $1,5 \mu \mathrm{g} / \mathrm{kg} / \mathrm{min}(27)$ & 12:01 & 96 & $100 / 68$ & $64-40$ & $79 / 52$ & $36 / 25(30)$ & - \\
\hline $2 \mu \mathrm{g} / \mathrm{kg} / \min (36)$ & 12:06 & 96 & $93 / 64$ & $69-39$ & $74 / 50$ & - & 2,3 \\
\hline $2,5 \mu \mathrm{g} / \mathrm{kg} / \mathrm{min}(45)$ & $12: 12$ & 100 & $98 / 62$ & $73-37$ & $74 / 56$ & - & 3,3 \\
\hline $3 \mu \mathrm{g} / \mathrm{kg} / \mathrm{min}(54)$ & $12: 17$ & 99 & $90 / 66$ & $74-34$ & $74 / 49$ & - & 4,1 \\
\hline
\end{tabular}

FC: frecuencia cardiaca; PA: presión arterial; PAM: presión arterial media

\section{Discusión:}

La HAP se caracteriza por presentar una remodelación vascular de la circulación pulmonar, predominantemente de arterias y arteriolas pulmonares distales, lo que provoca una mayor resistencia vascular pulmonar y, por ende, aumento de la presión de la arteria pulmonar ${ }^{9}$.
La inflamación juega un importante rol en la patogenia de la hipertensión pulmonar ${ }^{10} \mathrm{y}$ algunos estudios en HAP idiopática sugieren que las quimiocinas producidas en las arterias pulmonares de menor tamaño contribuyen al reclutamiento de células inflamatorias y a la proliferación de células musculares lisas en las arterias pulmonares ${ }^{11,12}$.

En los casos de pacientes portadores de VIH la presencia de HAP es independiente del recuento de linfocitos T CD4 (10) y el virus pasa a tener un rol relevante en la cascada inflamatoria debido a la exposición crónica a los productos virales, lo que sumado a la deficiencia de células $\mathrm{T}$ y a la producción alterada de citoquinas pro inflamatorias y factores de crecimiento, contribuyen a la disfunción vascular pulmonar y a la angioproliferación en los vasos pulmonares , factores claves en la patogenia de esta enfermedad ${ }^{10,13}$.

Los pacientes con VIH que desarrollan HAP tienen lesiones vasculares que son indistinguibles histológicamente de aquellos que tienen HAP idiopática ${ }^{14}$. Esto se basa en los hallazgos de células inflamatorias, incluidos macrófagos, linfocitos T y B y células dendríticas en las lesiones plexiformes encontradas en la HAP ${ }^{14}$. Las lesiones plexiformes pueden ser detectadas en el $78 \%$ de los pacientes con HTP-VIH ${ }^{15}$. Estas se caracterizan por obliteración luminal, disrupción de la íntima, hipertrofia de la túnica media, trombosis y, finalmente, recanalización del lumen ${ }^{16}$. Las principales proteínas virales que se asocian a la producción de las lesiones son la glicoproteína 120 y las proteínas Nef y Tat. ${ }^{10}$. Estas últimas poseen la capacidad de estimular monocitos y células endoteliales induciendo la angiogénesis 13. La glicoproteína 120, por su parte, es esencial para inducir apoptosis y aumentar la secreción de endotelina 1 en las células endoteliales ${ }^{13}$.

Los síntomas y signos asociados a HTP-VIH son similares a otros tipos de HAP. La disnea progresiva es el síntoma más común de presentación, seguido por edema de extremidades inferiores, tos no productiva, fatiga, sincope y dolor torácico ${ }^{17,18}$.El diagnóstico de HTPVIH requiere confirmar HAP, confirmar la infección por VIH y excluir otras posibles causas de hipertensión pulmonar ${ }^{19}$.Por lo anterior, se debe realizar ecocardiografía doppler transtorácica a estos pacientes, sobre todo si presentan disnea, para detectar signos indirectos de hipertensión pulmonar ${ }^{5,20}$. El criterio utilizado para el diagnóstico no invasivo de HAP es la presencia de una velocidad de jet de válvula tricúspide mayor a 2.8 $\mathrm{m} / \mathrm{s}$ o de un gradiente pico de insuficiencia de válvula tricúspide mayor a $30 \mathrm{mmHg}{ }^{21,22}$. En aquellos pacientes con sospecha clínica e imagenológica, es necesario 
realizar un estudio hemodinámico a través de una cateterización cardíaca derecha (CCD) para la confirmación diagnóstica de HTP-VIH, junto con establecer ausencia de alteraciones en cavidades cardíacas izquierdas ${ }^{5}$. En la CCD se incluyen los siguientes criterios diagnósticos para hipertensión pulmonar: presión media de arteria pulmonar (PAPm) mayor a $25 \mathrm{mmHg}$ en reposo o mayor a $30 \mathrm{mmHg}$ en ejercicio y presión de capilar pulmonar menor o igual a $15 \mathrm{mmHg}{ }^{20}$.

El test de vaso reactividad pulmonar al momento del $\mathrm{CCD}$, se utiliza para identificar aquellos pacientes que se beneficiarán del tratamiento con antagonistas de los canales de calcio ${ }^{5}$.

En el caso de los pacientes con HAP asociada a VIH no existe una utilidad demostrada en la realización del test de vaso reactividad al momento del $\mathrm{CCD}^{5,23}$. De realizarse, las características del vasodilatador a utilizar es que sea de rápida acción, seguro, fácil de administrar y con efectos sistémicos mínimos ${ }^{5}$. No existen estudios que avalen la eficacia de un fármaco sobre otro en este tipo de pacientes. En nuestra paciente a pesar de no haber una reversibilidad con el uso de vasodilatadores, si se constató un aumento en el gasto cardíaco.

En cuanto al tratamiento de HTP-VIH, este está menos determinado en comparación con otras formas de HAP $\left({ }^{5}\right)$. El uso de epoprostenol endovenoso, una prostaglandina con acción vasodilatadora, ha mostrado efectos positivos en la capacidad funcional y en los parámetros hemodinámicas recién descritos, en una serie pequeña de pacientes con HTP-VIH ${ }^{24}$. Otros estudios no controlados con prostaglandinas, como treprostinil subcutánea ${ }^{25} \mathrm{e}$ iloprost inhalado ${ }^{26}$ muestran esos mismos efectos en pacientes portadores de VIH. Un estudio aleatorio doble ciego que utilizó un antagonista selectivo de los receptores de la endotelina, ambrisentán, demostró seguridad y eficacia en el tratamiento de los pacientes sintomáticos con HAP ${ }^{27}$. Este estudio permitió la inclusión de pacientes con HTP-VIH, pero sólo representaron un 5\% de la muestra en estudio.

La anticoagulación se recomienda en los pacientes con HAP, pero los infectados por VIH a menudo tienen anormalidades en la hemostasia (ej. trombocitopenia) lo que puede aumentar el riesgo de hemorragias 28 En algunos reportes de casos clínicos se atribuye al sildenafilo una mejora de los síntomas y parámetros hemodinámicos en pacientes portadores VIH ${ }^{29,30}$, pero no hay estudios controlados, aleatorios, doble ciego que lo demuestren.

La mortalidad de pacientes con HIV-VIH se relaciona en forma importante a la hipertensión pulmonar propiamente tal, más que a otras alteraciones producidas por el VIH ${ }^{28}$ y la sobrevida disminuye significativamente en relación a mala capacidad funcional. A 3 años los pacientes con capacidad funcional I-II tiene un $84 \%$ de sobrevida en comparación con el $21 \%$ que tienen los pacientes con capacidad funcional III-IV ${ }^{28}$. Otros factores pronósticos relacionados a mejor sobrevida son el recuento de CD4 mayor a 212 células/ml, el uso de terapia antirretroviral combinada y la terapia con epoprostenol. ${ }^{28}$.

Finalmente, se ha demostrado que la terapia antirretroviral ha mejorado de manera significativa la sobrevida de los pacientes infectados con VIH, principalmente por la reducción de las infecciones oportunistas relacionadas con este agente ${ }^{31}$, sin embargo, la detección precoz de HTP-VIH es relevante para realizar el tratamiento correspondiente. Por lo tanto la recomendación es la búsqueda activa de HTP en todos los pacientes portadores de VIH.

\section{Referencias:}

1 SIRITHANAKUL A, MUBARAK K. Pulmonary arterial hypertension: newer treatments are improving outcomes, J Fam Pract 2004; 53:959-969.

2 SIMONNEAU G, ROBBINS I, BEGHETTI M, CHANNICK R, DELCROIX M, DENTON C. Up dated clinical classification of pulmonary hypertension. J Am Coll Cardiol. 2009; 54:43-54.

3 LEDERMAN M, SERENI D, SIMONNEAU G, VOELKEL
N. Pulmonary arterial hypertension and its association with HIV infection: an overview. AIDS 2008; 22: s1-s6.

4 NIRAV M, IJAZ K, RAJAL M, DOUGLAS S. HIV-Related pulmonary hypertension: analytic review of 131 cases. Chest 2000; 118:1133-1141.

5 GALIÈ N, HOEPER M, HUMBERT M, TORBICKI A, VACHIERY J, BARBERÀ J, et al. Guidelineson diagnosis and treatment of pulmonary hypertension: the task force on diagnosis and treatment of pulmonary hypertension of the european society 
of cardiology and of the European respiratory society. Eur Heart J. 2009;30:2493-537.

6 BARBARINIA G, BARBARO G. Incidence of the involvement of the cardiovascular system in HIV infection. AIDS. 2003;17 suppl 1:s46.

7 SITBONO,LASCOUX-COMBEC,DELFRAISSY J,YENIP, RAFFI F, DE ZUTTERED, et al. Prevalence of HIV-related pulmonary arterial hypertension in the current antiretroviral therapy era. Am J Respir Crit Care Med. 2008;177:108.

8 SPEICH R, JENNI R, OPRAVIL M, PFAB M, RUSSI E. Primary pulmonary hypertension in HIV infection. Chest. 1991;100:1268.

9 RUBIN LJ. Primary pulmonary hypertension. N Engl J Med 1997; 336:111-117.

10 HASSOUN P, MOUTHON L, BARBERÀ P, EDDAHIBI S, FLORES S, GRIMMINGER F, et. al. Inflammation, growth factors, and pulmonary vascular remodeling, Journal of the american college of cardiology, 2009; 54: s10-9

11 PERROS F, DORFMÜLLER P, SOUZA R, DURAND-GASSELIN I, GODOT V, CAPEL F, et al. Fractalkine-induced smooth muscle cell proliferation in pulmonary hypertension. Eur respir J 2007;29: 937-43.

12 SANCHEZ O, MARCOS E, PERROS F, FADEL E, TU L, HUMBERT M, et al. Role of endothelium-derived cc chemokine ligand 2 in idiopathic pulmonary arterial hypertension. Am J Respir Crit Care Med 2007;176:1041-7.

13 ALMODÓVAR S, CICALINI S, PETROSILLO N, FLORES D. Pulmonary hypertension associated with HIV infection pulmonary vascular disease: the global perspective. Chest 2010;137;6s-12s.

14 NICOLLS MR, TARASEVICIENE-STEWART L, RAI PR, BADESCH DB, VOELKEL NF. Autoimmunity and pulmonary hypertension: a perspective. Eur Respir J 2005; 26: 1110-8.

15 MEHTA NK, KHAN IA, MEHTA RN, SEPKOWITZ DA. HIV-related pulmonary hypertension: analytic review of $131 \mathrm{ca}-$ ses. Chest 2000; 118: 1133-1141.

16 MARECKI JC, COOL CD, PARR JE, BECKEY VE, LUCIW PA, TARANTALAF, et al. HIV-1 nef is associated with complex pulmonary vascular lesions in SHIV-nef-infected macaques. Am J Respire Crit Care Med 2006;174:437-45

17 MEHTA N, KHAN I, MEHTA R, SEPKOWITZ M. HIV-related pulmonary hypertension Analytic review of 131 cases. Chest 2000; 118:1133-1141.

18 ZUBER JP, CALMY A, EVISON JM, HASSE B, SCHIFFER V, WAGELS T, et.al. Pulmonary arterial hypertension related to HIV infection: improved hemodynamics and survival associated with antiretroviral therapy. Clin Infect Dis. 2004;38:1178.

19 GALIÈ N, HOEPER M, HUMBERT M, TORBICKI A, VA-
CHIERY JL, BARBERÁ JA, et.al. Guía de práctica clínica para el diagnóstico y tratamiento de la hipertensión pulmonar. Rev Esp Cardiol. 2009; 62 12: 1464.e1-e58.

20 BADESCH DB, CHAMPION HC, SANCHEZ MA, HOEPER MM, LOYD JE, MANES A, et al. Diagnosis and assessment of pulmonary arterial hypertension. J Am Coll Cardiol. 2009; 54: s55.

21 ELSTEIN D, KLUTSTEIN MW, LAHAD A, ABRAHAMOV A, HADAS-HALPERN I, ZIMRAN A. Echocardiographic assessment of pulmonary hypertension in gaucher's disease. Lancet 1998; 351: $1544-6$

22 MCQUILLAN BM, PICARD MH, LEAVITT M, WEYMAN AE. Clinical correlates and reference intervals for pulmonary artery systolic pressure among echocardiographically normal subjects. Circulation 2001;104: 2797-802.

23 ESCRIBANO P, BARBERÀ MIR JA, SUBERVIOLACV.Current diagnostic and prognostic assessment of pulmonary hypertension. Rev Esp Cardiol. 2010;63:583-96.

24 AGUILAR RV, FARBER HW. Epoprostenol (prostacyclin) therapy in HIV- associated pulmonary hypertension. Am J Respir Crit Care Med 2000; 162:1846-1850.

25 CEA-CALVO L, ESCRIBANO P, TELLO M, LAZARO M, GOMEZ M, DELGADO J, et. al. Tratamiento de la hipertensión pulmonar asociada a la infección por VIH con treprostinil. Rev Esp Cardiol 2003;56:421-425.

26 GHOFRANI HA, FRIESE G, DISCHER T, OLSCHEWSKI H, SCHERMULY R, WEISSMANN N, et al. Inhaled iloprostis a potent acute pulmonary vasodilator in HIV-related severe pulmonary hypertension. Eur Respir J 2004; 23:321 - 326.

27 GALIÈ N, OLSCHEWSKI H, OUDIZ RJ, TORRES F, FROST A, GHOFRANI HA, et al. Ambrisentan for the treatment of pulmonary arterial hypertension. Results of the ambrisentan in pulmonary arterial hypertension, randomized, double-blind, placebo-controlled, multicenter, efficacy (ARIES) study 1 and 2. Circulation 2008;117:3010 - 3019.

28 NUNES H, HUMBERT M, SITBON O, MORSE JH, DENG $\mathrm{Z}$, KNOWLES JA, et al. prognostic factors for survival in human immunodeficiency virus-associated pulmonary arterial hypertension. AmJj Respir Crit Care Med. 2003;167:1433.

29 CARLSEN J, KJELDSEN K, GERSTOFT J. Sildenafil as a Successful treatment of other wise fatal HIV-related pulmonary hypertension. AIDS 2002;16:1568.

30 SCHUMACHER YO, ZDEBIK A, HUONKER M, KREISEL W. Sildenafil in HIV-related pulmonary hypertension. AIDS 2001;15:1747.

31 Joint united nations programe on HIV/AIDS (UNAIDS) and world health organizations (WHO). AIDS Epidemic update 2009. Geneva, Switzerland: WHO; 2009. 